\title{
BILDJOURNALISMUS
}

\section{Hässlichkeit zu zeigen bedarf keiner digitalen Politur}

\author{
Der Fotograf Till Mayer arbeitet mit Hilfsorganisationen \\ in Kriegs-, Krisen- und Katastrophengebieten zusammen.
}

$\mathrm{I}$ ch glaube, ich habe es als Teenager in einem politischen $\mathrm{Ab}$ reißkalender gelesen. Während der äthiopischen Dürrekatastrophe, Mitte der 1980er Jahre, soll ein amerikanisches Fotografenteam sterbende Kinder mit Asche geschminkt haben. So sollten die Bilder eindrücklicher wirken. Ich habe diese kurze Meldung nie vergessen. Sie hat mich schockiert. Schon mit 16 wollte ich einmal das tun, was ich heute mache. Fotografen waren für mich genauso Helden wie die Guerilleros Lateinamerikas, jene Kämpfer gegen Unterdrückung und Diktatur, die sie ablichteten. Und dann das?!

Im Jahr 2000 fotografierte ich selber die Folgen einer Dürre in dem afrikanischen Land. Die Ausmaße der Katastrophe waren bei weitem nicht so tiefgreifend wie die, deren Bilder knapp 15 Jahre zuvor die Welt bewegten. Es war grausam genug. Menschen im 21. Jahrhundert an Hunger leiden und auch sterben zu sehen, ist unfassbar. Kinder blickten mich an, mit Augen von alten Menschen. Die Körper so ausgezehrt, dass die Köpfe überproportional groß wirkten.

Ich war mit Helfern des „Roten Kreuzes“ unterwegs. Auf der Suche nach Nomadendörfern, die auf keiner Karte standen. Ansammlungen von Hütten, irgendwo verloren in der staubigen Weite des Ogaden. Manchmal steckten die Menschen verdorrte Kuhkadaver in die kahlen Bäume und Büsche. Bizarre und verzweifelte Hilferufe. Mit den Tieren starb auch die zukünftige Lebensgrundlage. In einem Dorf reihten sie die Opfer des Hungers vor mir auf. Mit einer Schubkarre brachten sie eine sterbende Alte. Ein halbes Dutzend Kinder mühte sich aufgereiht aufrecht zu sitzen. Sie weinten vor Schmerzen, als ihnen die Eltern ihre Hemden auszogen, damit ich ihre ausgemergelten Körper sehe.

Damit ich sie fotografiere. Jeden einzelnen von ihnen. Ihre Eltern konnten sich noch erinnern: Damals vor 15 Jahren, ka- 
Äthiopien 2000:

Kind bei einem

Versorgungszentrum

während einer

schweren Dürrephase

alle Fotos in diesem Beitrag von Till Mayer

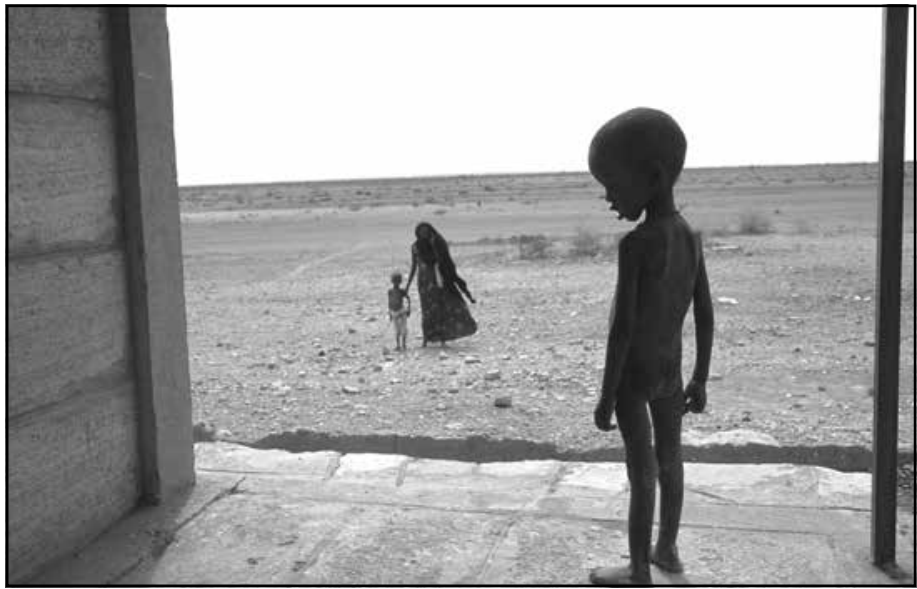

men auch Männer mit kleinen schwarzen Kästen. Und dann Hilfe. Also ließen sie mich nicht gehen, bevor ich nicht bei jedem einmal den Auslöser gedrückt hatte. Ich habe diese Bilder nie verwendet. Die anderen Fotos waren schon traurig genug. Aber diese Szene hat mich betroffen gemacht. Manchmal denke ich mir heute, wie ein Fotograf dann noch Hungernde und Sterbende schminken kann.

Es macht mich immer noch fassungslos. Nach Äthiopien nahm ich mir für ähnliche Aufträge ein Jahr Auszeit. Es war notwendig. Dass es dennoch wichtig war, das Leiden zu dokumentieren, stellte und stelle ich nicht in Frage. Die Welt soll wissen, was da passiert. Ungeschminkt.

Eines der Fotos, das ich in Äthiopien für das „Rote Kreuz“ machte, lief sehr gut. Es landete in vielen Zeitungen weltweit und später in Büchern und Ausstellungen. Ein nackter Junge steht da. Abgemagert, die Haut ledrig, der Kopf monströs groß. Im Hintergrund Steppe und eine Frau, die dem Jungen entgegenkommt. Sie stützt ihr Kind, damit es vor Schwäche nicht fällt. Das Bild entstand in der Nähe eines Versorgungszentrums für die Dürrebetroffenen.

Bei einer Ausstellung beschwerte sich eine ältere Dame bei mir, dass ich das Kind nackt fotografiert habe. „Aber so war es doch. Das Kind hatte gar nichts mehr außer seinem nackten Leben." Ich überzeugte die Frau nicht. Viele Hilfsorganisationen geben ihren Fotografen bei einem Auftrag einen mehrseitigen Ethik-Leitfaden zum Unterschreiben. Das ist gut so. Basics stehen dort: Das Einverständnis des Portraitierten einzuholen, zum Beispiel. Das oben beschriebene Bild hätte ich demnach meist nicht machen können. Nackte Kinder sind laut Leitlinien tabu. 


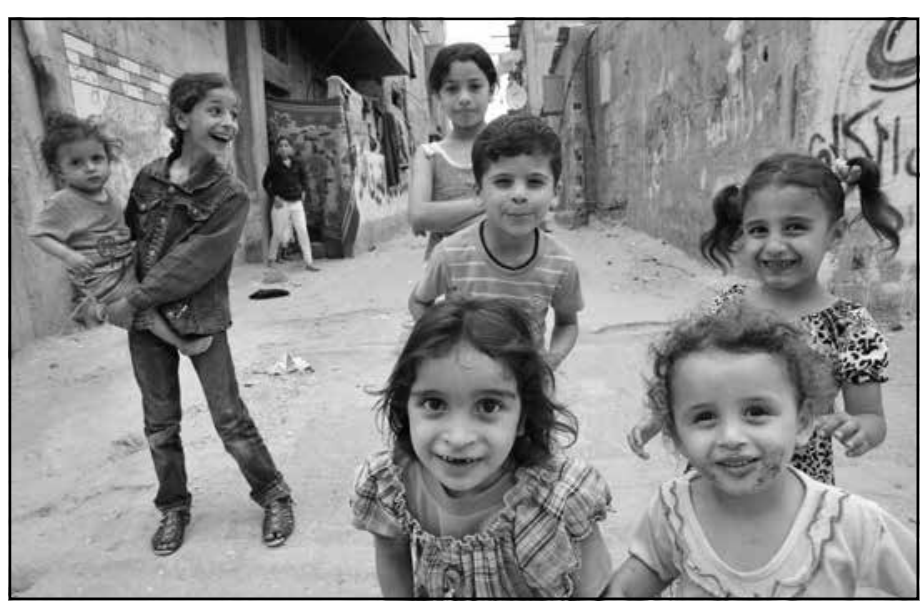

Wie fotografiere ich ethisch in einer Umgebung, in der oft jede Ethik nicht mehr zählt? Wie zeige ich die Grausamkeit eines Krieges, die Folge einer Katastrophe, ohne den Opfern die Würde zu nehmen? Ein guter Fotograf hat Respekt. Respekt den Menschen gegenüber, die er portraitiert. Respekt der Aufgabe gegenüber, der er sich stellt.

Ich mache mir nichts vor: Meine Arbeit schmerzt nicht selten die Menschen, die ich vor meiner Kamera habe. Sie erzählen mir, einem Fremden, ihre Lebensgeschichte. Nicht selten sind es unfassbare Schicksale. Nach dem Interview hole ich meine Kamera hervor. Wenn ich gehe, bleiben die Interviewpartner meist kraftlos zurück. Weil sie nicht selten alles gegeben haben, um mir ihr Schicksal zu erzählen. Doch das ist ihnen wichtig. Dass ihre Geschichte gehört wird.

Wie die Geschichte von Frau Trung Thi Thuy, Mutter von vier mutmaßlichen „Agent Orange“-Opfern. Zwei ihrer Söhne liegen Tag für Tag auf einer Pritsche. Ihre Gliedmaßen sind verkrümmt. Frau Thuy rackert den ganzen Tag auf dem Feld, und in der Pause eilt sie zu ihren schwerst behinderten Kindern, um sie zu versorgen.

Mit dioxinhaltigen Herbiziden wollten die US-amerikanischen Streitkräfte während des Vietnamkriegs den Urwald entlauben und so den nordvietnamesischen Verbänden die Rückzugsmöglichkeiten nehmen. Zurück bleibt Jahrzehnte nach Kriegsende ein Heer von schwerstbehinderten „Agent Orange“Opfern. Das Gift verändert auch das Erbgut.

Familie Thuy ist betroffen. Zu wissen, dass das eigene Schicksal so schwer ist, dass selbst ein Journalist aus dem fernen Europa darüber berichtet: Für Frau Thuy muss das sehr schmerz-
Gaza 2013:

Ihre Familien wurden vor über sechs Jahrzehnten vertrieben - das Flüchtlingselend dauert über Generationen hinweg. 
Kongo 2013/2014:

Flüchtlingslager

in Goma

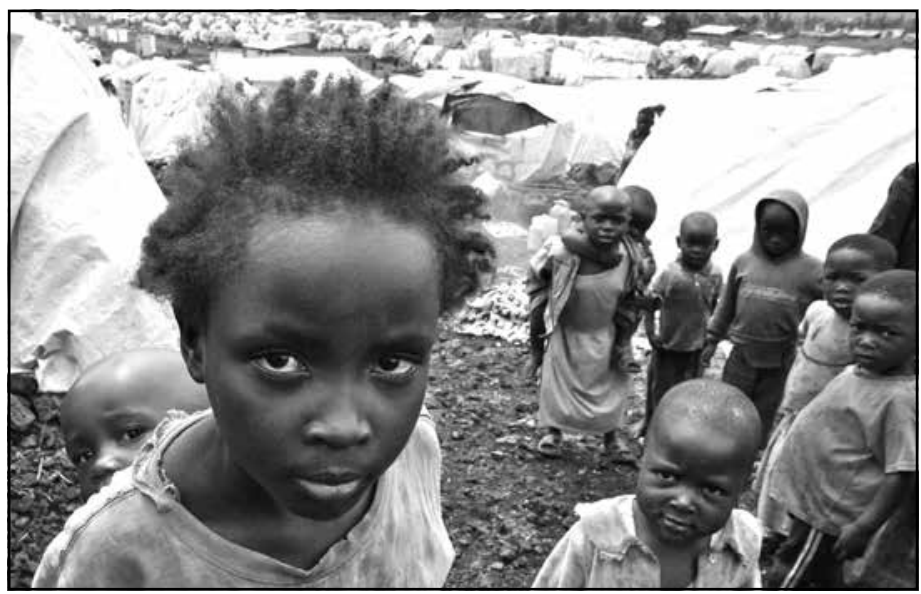

haft gewesen sein. Aber, dass Unrecht bekannt wird, ist auch einer Bauersfrau in Vietnam wichtig. Bis heute wartet sie auf eine Entschädigung, auf eine Entschuldigung der amerikanischen Regierung für ihre Giftabwürfe. Mit meinen Fotos der Familie Thuy wollte ich nicht deformierte Knochen zeigen oder Hoffnungslosigkeit vorführen. Aber eine Familie portraitieren, die kämpft. Eine Mutter, die sich kümmert. Den Fokus auf das Gesicht, die Augen zu legen, hilft dabei, den Menschen zu zeigen und nicht den Mittelpunkt auf verdrehte Gliedmaßen zu setzen.

Ich habe kein Mitleid mit Frau Thuy. Sie ist ein Mensch, von dem ich lernen kann. Eine Frau, die ich für ihre Tapferkeit bewundere. Das will ich mit meinen Bildern erzählen. Auch wenn ich dabei die Missbildungen ihrer Kinder zeige, es geht nicht darum zu beschönigen.

Ethische Fotografie bedeutet Respekt, Respekt und nochmals Respekt. Portraitierte nicht zu Haut und Knochen zu degradieren. Sie nicht als hilflose Opfer darzustellen. Das ist mir wichtig. Manchmal ist das nicht zu schaffen, wie bei der ausgehungerten alten Frau, die man mit einem Schubkarren zu mir schob. Das war ein Augenblick, bei dem ich auch als Fotograf nur noch Hoffnungslosigkeit gesehen habe.

Immer wieder hört man die Klagen: „Bei all den schlimmen Bildern muss man doch abstumpfen..." Aber sehen wir wirklich so oft, wie ein Mensch unter einem Krieg leidet? Wir sehen tagelang Rauchschwaden in Kobane aufsteigen. Flüchtlinge, die in Massen strömen. Gezielte Nato-Luftschläge. Aber Reportagen, die uns die betroffenen Menschen, ihr Schicksal, wirklich nahebringen? Das ist dann doch eher überschaubar. Und stumpfen diese Fotos und Reportagen wirklich ab? 


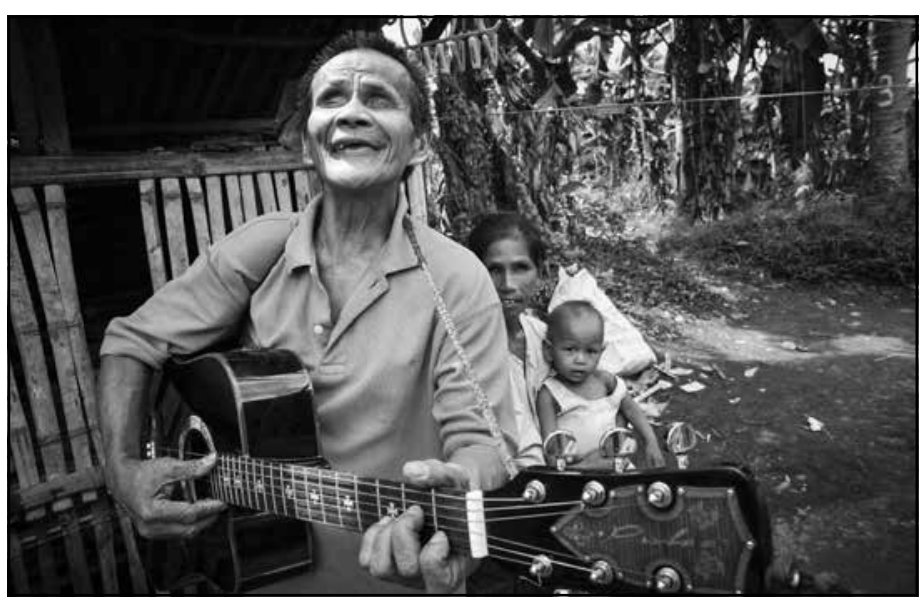

Den „World Press Photo Award“ 2013 holte ein Foto, das in Gaza entstand. Mitten in einer Menge hält ein Vater sein bei einem israelischen Angriff getötetes Kind. Was für ein starkes, trauriges und erschütterndes Foto. Was für ein grausamer Augenblick. Warum der Fotograf meinte, das Bild so sehr zu bearbeiten, dass es glatt wie aus einem Kinofilm wirkt - ich habe es nicht verstanden. ${ }^{1}$ Einem respektvoll gemachten Bild wurde so ein Stück Seele genommen. Weil versucht wurde, das Bild perfekt zu gestalten. Doch im Krieg ist nichts perfekt - außer, nicht

12013 erhielt der Schwede Paul Hansen den "World Press Photo Award“ für das oben beschriebene Bild, das er im November 2012 für die Tageszeitung "Dagens Nyheter" in Gaza-Stadt aufgenommen hatte. Nach der Auszeichnung wurde ihm vorgeworfen, das Bild mit Photoshop bearbeitet zu haben - die Lichtverhältnisse seien nahezu perfekt und entsprächen überdies nicht dem Stand der Sonne im November, so lauten die Vorwürfe. (vgl. Katzenberger, Paul (2013): Zu viel Licht. In: Sueddeutsche.de vom 14.5. www.sueddeutsche.de/medien/streit-ueber-world-press-photo-zu-viel-licht-1.1672199

(zuletzt aufgerufen am 12.11.2014) und Haug, Kristin (2013): World Press Photo Award: „Schwer zu sagen, wo Betrug anfängt. In: Spiegel-Online vom 18.5. www.spiegel.de/kultur/gesellschaft/jenskriese-ueber-manipulation-paul-hansens-und-world-press-photoaward-a-900383.html (zuletzt aufgerufen am 12.11.2014)). Es entwickelte sich eine intensive Debatte über die Grenzen der Bildbearbeitung. Hansen hat die Bearbeitung eingeräumt, die Vorwürfe der Fälschung aber zurückgewiesen. (vgl.: o. A. (2013): World Press Photo Award: Streit ums Leid-Bild. In: SpiegelOnline vom 14.5. www.spiegel.de/kultur/gesellschaft/world-press-photoaward-paul-hansen-soll-photoshop-verwendet-haben-a-899807.html (zuletzt aufgerufen am 12.11.2014)).
Philippinen 2014, ein Jahr nach dem Taifun Haiyan/Yolanda: Mit seiner Gitarre ernährt der blinde Oscar Borer seine Kinder. Für sich und die anderen Katastrophenopfer hat er ein Lied geschrieben - eine einfache Weihnachtsweise. „Das gibt jetzt den Taifun-Opfern am meisten Hoffnung." 


\section{Philippinen 2014, ein Jahr nach dem Taifun \\ Haiyan/Yolanda: Mit Hilfe der Organisa- tion „Handicap Interna- tional" konnte sich der junge Mann eine neue Hütte bauen. Die meis- ten Arbeiten konnte er trotz seiner Behinde- rung selbst erledigen. Als Dankeschön gibt es einen Handstand.}

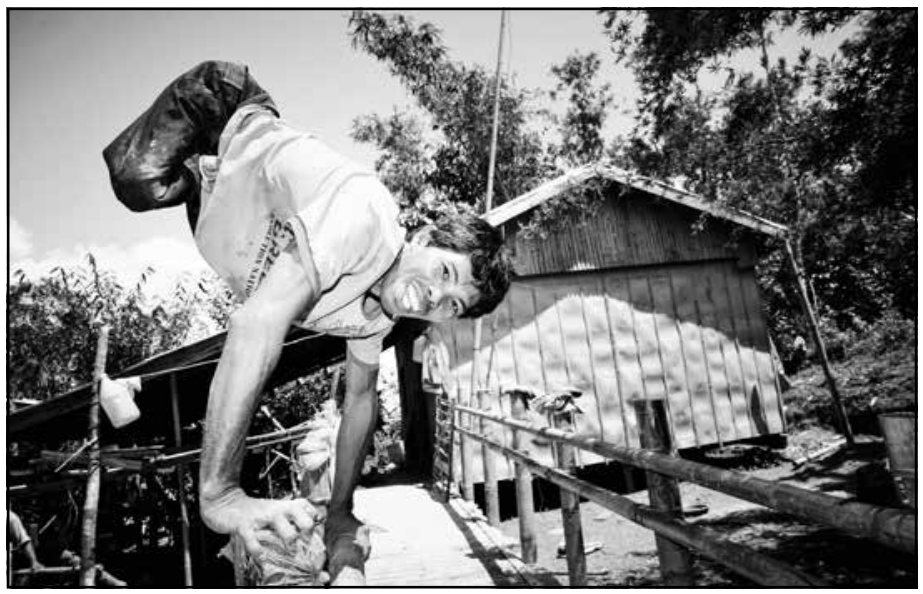

selten, die Tötungsmaschinerie. Um seine Hässlichkeit zu zeigen, bedarf es keiner digitalen Politur.

Das Bild liegt im Trend, die Medienwelt wird glatter. Und schneller. Seit einem Buch- und Ausstellungsprojekt über KZÜberlebende in der Ukraine im Jahr 2007 engagiere ich mich für verarmte Rentner in dem osteuropäischen Land. So ist ein kleines Hilfsprojekt entstanden - und über Jahre Vertrauen. Dadurch wiederum Bilder, die ich sonst nicht hätte machen können. Türen und Interviewpartner öffneten sich mir. Menschen sprachen mit mir, weil ich einen Fürsprecher hatte. Der ihnen versicherte, dass ich meine Interviewpartner mit Respekt behandle. Deswegen waren sie bereit, sich fotografieren zu lassen. In einer Armut, die sie als beschämend empfanden.

Langfristig angelegte Recherchen und Fotoprojekte - welche Redaktion kann sich das noch leisten? Aber gerade sie verhindern eine einseitige Sicht in einer Welt, die scheinbar immer komplexer wird.

Ethische Fotos erklären, erzählen. Sie reißen mit. Sie können Grausamkeit spiegeln, bestürzen und verzweifeln lassen. Sie können Tod und Hunger, aber auch Hoffnung mitten im Chaos zeigen. Sie haben immer eines gemeinsam - Respekt, vor dem, was uns alle einen sollte: Menschlichkeit.

\section{Zur Person}

Till Mayer arbeitet seit vielen Jahren als Journalist und Fotograf eng mit dem „Roten Kreuz“/,Roten Halbmond“, „Handicap International" und anderen Hilfsorganisationen zusammen. Er bereiste zahlreiche Kriegs- und Krisenländer sowie Katastrophengebiete Afrikas, Asiens und Europas. Seine Aufgabe als Informationsdelegier- 


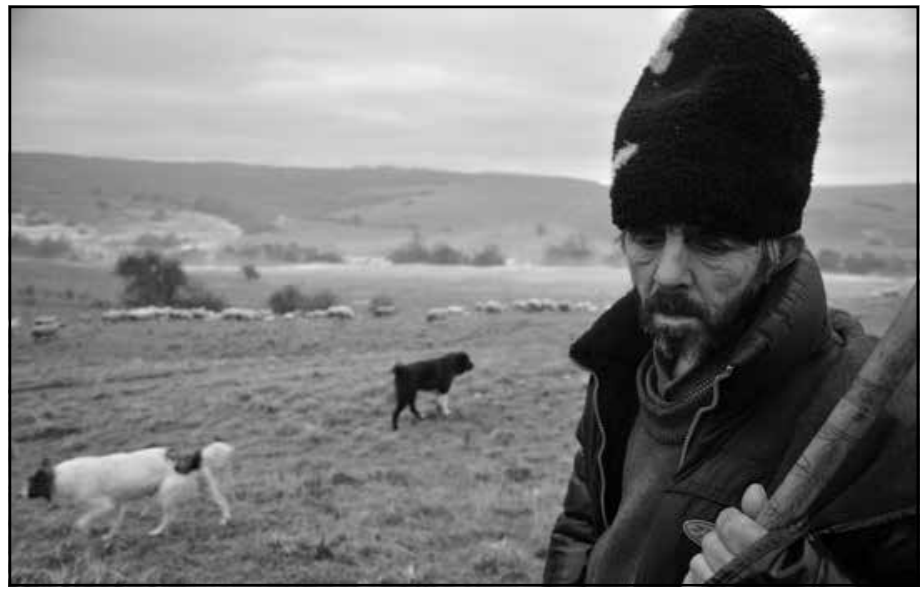

ter des Internationalen bzw. „Deutschen Roten“ Kreuzes führte ihn auf den Balkan, in die Türkei, nach Sri Lanka, in den Irak und den Iran. Ehrenamtlich engagiert sich Till Mayer bei den „Rotkreuz“Kreisverbänden Bamberg und Lichtenfels (Bayern) als Vorstandsmitglied und Konventionsbeauftragter. In seiner Heimat hat er die Leseraktion „Helfen macht Spaß“ aufgebaut, die in Zusammenarbeit mit dem „Roten Kreuz“ und anderen Wohlfahrtsverbänden Bedürftige unterstützt. Ehrenamtlich engagiert er sich für bedürftige Senioren in Lviv (Ukraine). Bei der Tageszeitung „ObermainTagblatt“ ist er als Redakteur angestellt. Als freier Fotograf und Journalist arbeitet er für „Spiegel-Online“ sowie zahlreiche Zeitungen und Magazine. Seine Fotos werden weltweit in Ausstellungen gezeigt. Er ist Autor von mehreren Bildbänden. Für sein humanitäres Engagement als Journalist wurde er mehrfach ausgezeichnet. Mehr im Internet: www.tillmayer.de

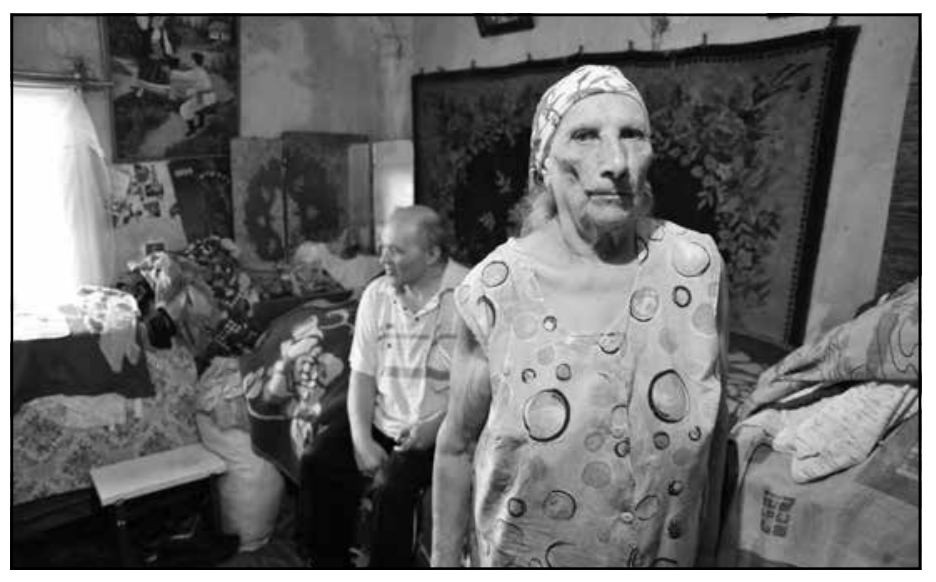

Rumänien 2011:

Zeitreise mitten in

Europa - ein Schafhirte in Siebenbürgen.

Ukraine 2014:

Ein altes Ehepaar

in Lviv (Lemberg) -

aufgrund des Konflikts steigt die Inflation und die Kaufkraft der Rente sinkt. Das Ehepaar muss mit weniger als 150 Euro im Monat überleben - für Essen, Medikamente, Heizen, Kleidung. Oft gehen die beiden hungrig ins Bett. 